\title{
Modelling strategies for semiconductor ring lasers
}

\author{
Antonio Pérez S. ${ }^{1}$, Sandor Fürst ${ }^{2}$, Alessandro Scirè ${ }^{1}$, Julien Javaloyes ${ }^{3}$, Salvador Balle ${ }^{3}$ and \\ Marc Sorel ${ }^{2}$ \\ ${ }^{1}$ Instituto de Física Interdisciplinar y Sistemas Complejos, IFISC, CSIC-UIB, Campus UIB, \\ Ctra. Valldemossa km. 7.5, E-07122 Palma de Mallorca, Spain. \\ ${ }^{2}$ Dept. of Electronics and Electrical Engineering, University of Glasgow, 1 Lilybank Gdns \\ Glasgow, G12 8RZ, United Kingdom. \\ ${ }^{3}$ Instituto Mediterráneo de Estudios Avanzados, IMEDEA, CSIC-UIB, Ctra. Valldemossa km. \\ 7.5, E-07122 Palma de Mallorca, Spain.
}

\begin{abstract}
We have analyzed experimentally and theoretically the modal properties of a semiconductor ring laser and the wavelength jumps that occur in connection with directional switching above threshold. A transfer matrix analysis allow us to explain the transfer function measurements when amplified spontaneous emission in the cavity is accounted for. Moreover the transfer matrix analysis permits to determine the threshold condition for the laser modes, which split in two branches due to the symmetry breaking imposed by the output coupler and output waveguides. The wavelength jumps displayed by the device above threshold are interpreted with the frequency splitting and threshold difference between these two branches of solutions, together with the redshift of the material gain.
\end{abstract}

Keywords: Semiconductor lasers, ring lasers, modal properties, coupled cavities, laser dynamics

\section{INTRODUCTION}

For many years, Semiconductor Ring Lasers (SRLs) have attracted interest for their potential in the fabrication of micro-cavity lasers and densely integrated photonics circuits. It has recently been realized that the coexistence of two counterpropagating modes in the same gain medium leads to a large variety of operating regimes and dynamics that are profoundly different from those of Fabry-Perot lasers. ${ }^{1}$ One of the most interesting property is the extremely fast directional bistability that opens up a new scenario in the development of all-optical memories and signal processing. ${ }^{2,3}$ Besides their switching characteristics, SRLs show several unexpected behaviors such as hysteresis in the lasing direction ${ }^{4,5}$ and atypical lasing mode selection rules. In particular, when current or temperature are changed, the lasing mode does not hop between consecutive cavity modes but exhibits sudden jumps between several cavity modes only when the lasing direction reverses. This characteristic strongly enhances the stability of the lasing wavelength against changes in the operating conditions.

We measure the transfer function of SRL devices in the frequency domain, which provides us with a map of the cavity resonances, and the emission wavelength of the SRLs when biased above threshold. The transfer function can be theoretically explained by considering the perturbation induced by the output couplers, which induces a symmetry breaking in the resonant cavity and a modulation of the cavity losses. For the geometry considered, the cavity losses have a wavelength periodicity that corresponds to three ring cavity modes, which explains the measured hops in wavelength as the bias current of the laser is increased.

\section{ANALYSIS}

The device layout consists of a ring cavity with a ring radius of $300 \mu \mathrm{m}$, coupled to a straight output waveguide by a point evanescent coupler. The waveguides are $2 \mu \mathrm{m}$ wide and the gap between the ring and the output waveguides is $750 \mathrm{~nm}$, providing a theoretical coupling ratio of $12 \%$. To minimize the backreflections, the output waveguides are $10^{\circ}$ tilted to the cleaved facets.

Email: antonio@ifisc.uib.es 


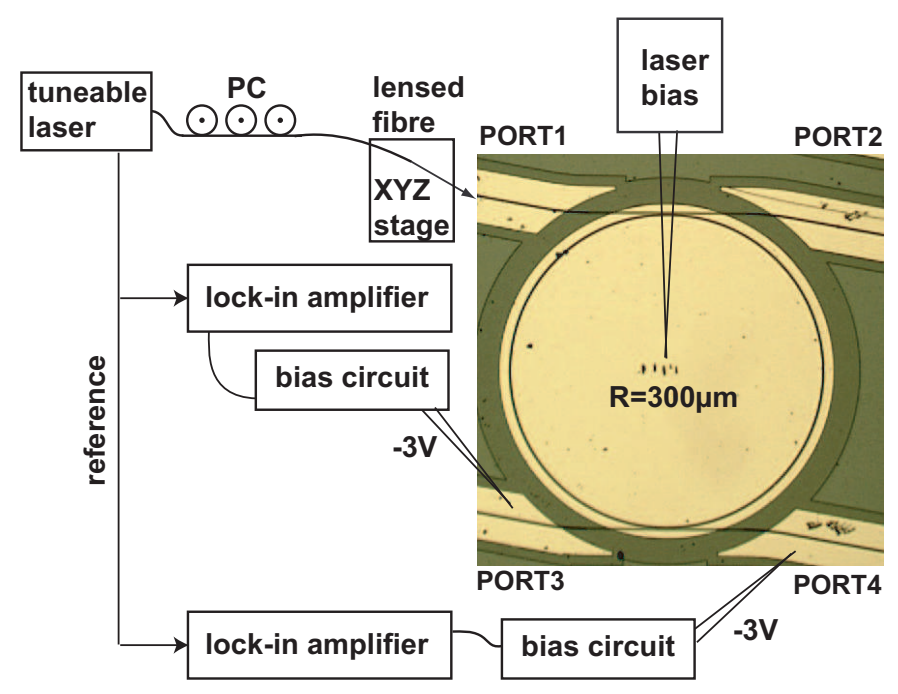

Figure 1. Optical micrograph of a $300 \mu \mathrm{m}$-radius ring laser with the corresponding measurement setup.

The wafer used to fabricate the devices is a multiple quantum well AlGaInAs/InP structure, grown by metalorganic chemical vapor deposition (MOCVD). The waveguides were defined by electron beam lithography and transferred to a PECVD (Plasma Enhanced Chemical Vapor Deposition) $\mathrm{SiO}_{2}$ layer, using $\mathrm{CHF}_{3}$ reactive ion etching (RIE). A shallow etched ridge-waveguide was then defined by RIE, using a chemistry of $\mathrm{CH}_{4} / \mathrm{H}_{2} / \mathrm{O}_{2}$ process, which is selective to the $\mathrm{Al}$ containing core layer and thus ensures a very good control over the etching depth and the power coupling ratio. The etching depth provides an effective refractive index difference of $\Delta n=0.064$ which makes the bending losses negligible down to ring radii of $140 \mu \mathrm{m}$. The subsequent deposition of a $\mathrm{SiO}_{2}$ layer was followed by contact window definition for current injection. Finally, metal contacts were deposited on both the epitaxial and substrate sides of the wafer section.

In order to analyze the cavity resonances of the SRL, we inject through port \#1 a monochromatic field from a tunable laser and we measure the photo-current generated in ports \#3 and \#4, which are reverse biased, as shown in Fig. 1. Feedback levels from unbiased and biased outputs were estimated to be $-79.2 \mathrm{~dB}$ and -94.6 , respectively. During these measurements, the ring is biased close to transparency to minimize the losses. Fig. 2 shows the power collected at ports \#3 and \#4 as the input wavelength is scanned. It can be seen that the power in port \#3 displays narrow and well defined peaks at wavelengths equispaced by $0.4 \mathrm{~nm}$. The peak heights show the expected profile defined by the wavelength-dependent gain spectrum in the structure but also an additional modulation that occurs every three longitudinal modes. A zoom around these peaks (in Fig. 3) reveals that they possess a doublet structure, with the splitting between the two subpeaks being of the order of $4 \mathrm{GHz}$. The power collected at port \#4 presents a similar structure with the same periodicity, but instead of displaying peaks above a spontaneous-emission noise background, it shows dips on such a background.

When the laser is biased above threshold, the main lasing direction does not remain stable for all current values. Fig. 4 reports the L-I curve of a $300 \mu m$ radius device, showing the typical SRL switching behaviour between the $\mathrm{CW}$ and $\mathrm{CCW}$ directions for increasing current, ${ }^{5}$ along with the wavelength of the main lasing direction. It clearly appears that, in the regime of directional switching, the dominant lasing wavelength remains constant (except for a small thermal drift) when the lasing direction does not hop, but it suddenly jumps by three cavity modes when the lasing direction reverses. Fig. 6 show the measured detuning between doublets showing a modulation of the peaks that roughly corresponds to a periodicity of 3 peaks.

In order to explain the previous results for the power at ports \#3 and \#4, the transfer matrix of the complete SRL structure ${ }^{7}$ can be computed. For the sake of simplicity we assume that the two couplers are lossless and equal and that there is no reflectivity from the output facets \#3 and \#4, since the corresponding output waveguides are reverse biased. When a field of amplitude $E_{1}$ and frequency $\omega$ is injected through port \#1, the fields $\mathrm{CW}$ 


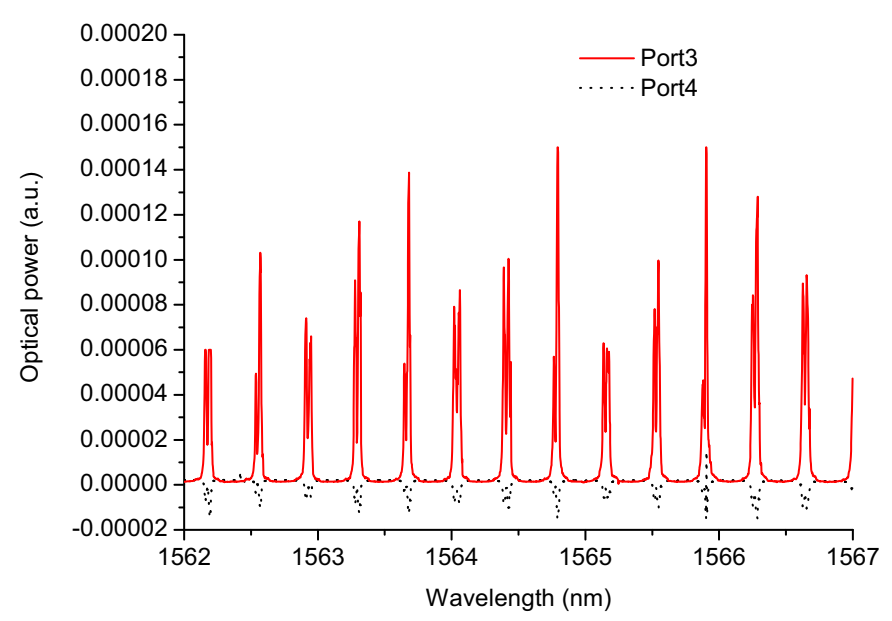

Figure 2. Detected power at port \#3 and \#4.

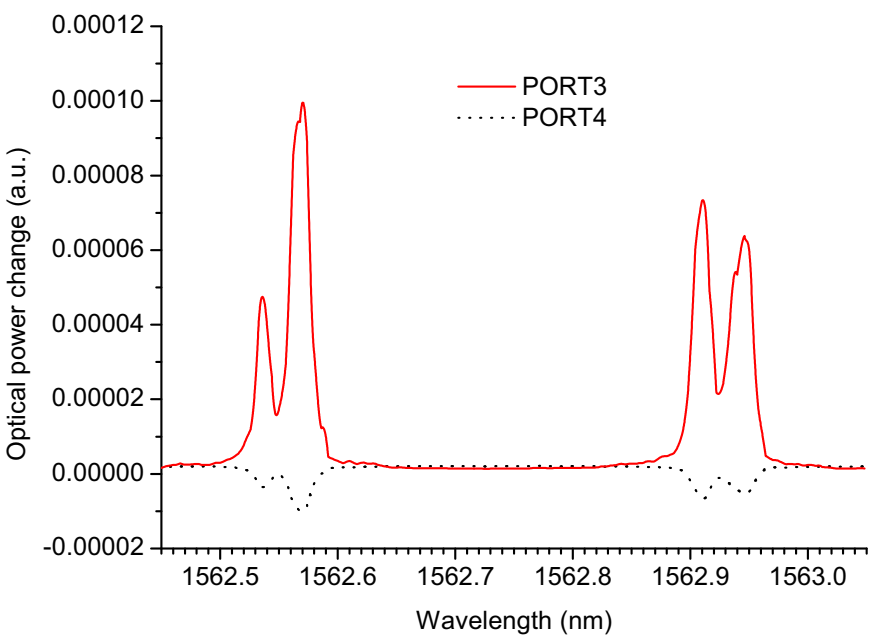

Figure 3. Zoom in of the measured lines.

$\left(E_{+}\right)$and $\mathrm{CCW}\left(E_{-}\right)$are given by

$$
\begin{aligned}
{[1-} & \left.-\left(1-\delta^{2}\right) m(\omega)-\left(1-\delta^{2}\right)(1-m(\omega)) e^{i \theta}\right] E_{+} \\
& +\delta^{2} \sqrt{1-\delta^{2}} \hat{r}_{1} e^{i \theta} E_{-}=i \delta \hat{t}_{1} E_{i} \\
{[1 \quad-} & \left.\left(1-\delta^{2}\right) m(\omega)-\left(1-\delta^{2}\right)(1-m(\omega)) e^{i \theta}\right] E_{-} \\
& +\delta^{2} \sqrt{1-\delta^{2}} \hat{r}_{2} e^{i \theta} E_{+}=i \delta \sqrt{1-\delta^{2}} \hat{r}_{2} \hat{t}_{1} E_{i} .
\end{aligned}
$$

In these equations, $\hat{r}_{1(2)}=r_{1(2)} e^{i \omega\left(n_{1(2)} L_{1(2)}+n_{c} L_{c}\right) / c}, \hat{t}_{1}=t_{1} e^{i \omega\left(n_{1} L_{1}+n_{c} L_{c}\right) / c}$ and $m(\omega)=\hat{r}_{1} \hat{r}_{2}$, where $r_{1(2)}, n_{1(2)}$, $L_{1(2)}$ are the reflectivity of the facets at port \# 1(2), and the effective index and length of waveguide \# 1(2), respectively, $t_{1}$ is the transmittivity of port $\# 1$, and $n_{c}$ and $L_{c}$ stand for the effective index and length of the coupler waveguides. In addition, $\delta^{2}$ is the coupling efficiciency of the coupler, and $\theta=q_{R} L_{R}+2 \omega n_{c} L_{c} / c$, where $L_{R}$ is the length of the pumped sections of the SRL, where the propagation constant for the optical field is $q_{R}$, given by

$$
q_{R}^{2}=\frac{\omega^{2}}{c^{2}}\left[n_{R}^{2}(1-\Gamma)+\Gamma \chi(\omega, N)\right],
$$

where $c$ is the speed of light in vacuo, $n_{R}$ is the effective refractive index of the SRL waveguide, $\Gamma$ is the optical confinement factor of the field to the active region of the SRL, and $\chi(\omega, N)$ is the frequency-dependent optical 


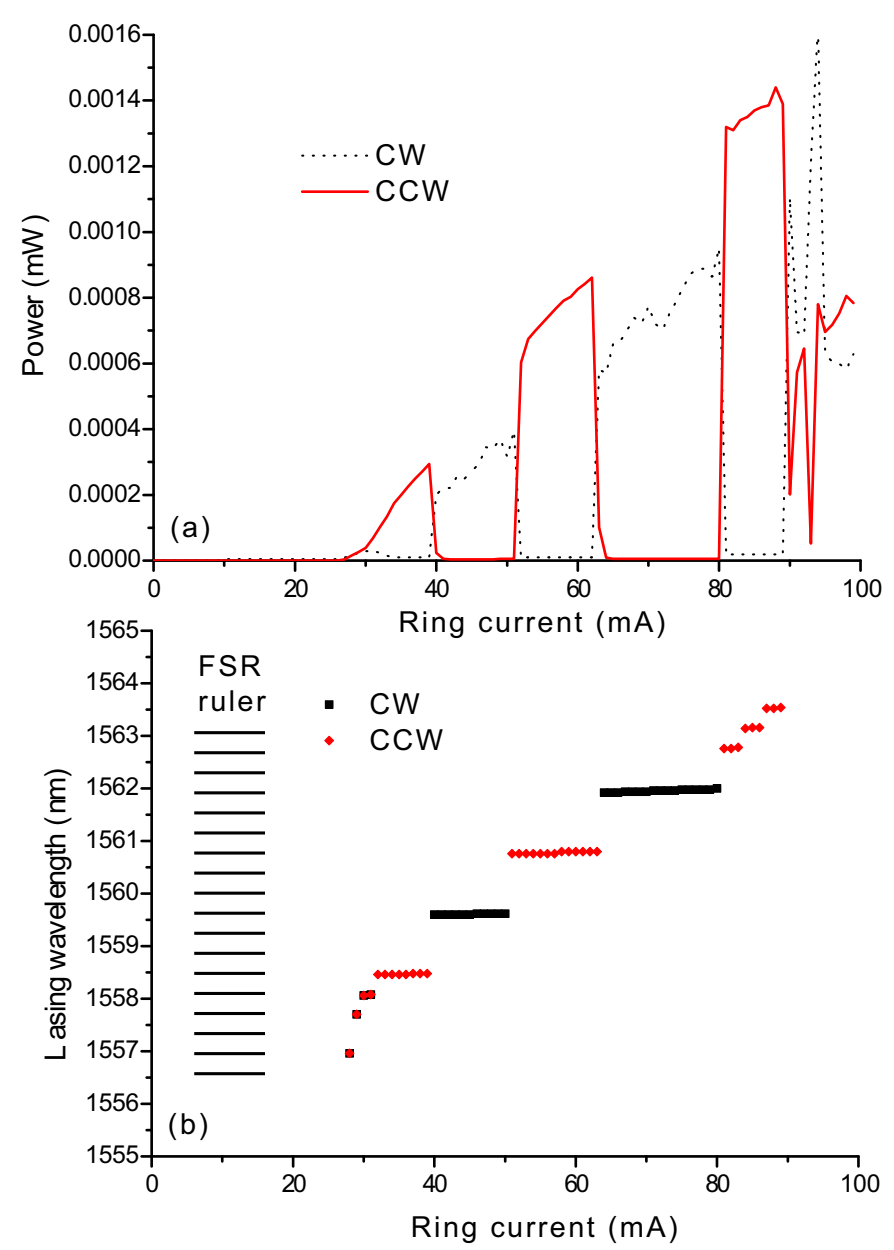

Figure 4. LI curve (a) and peak wavelength map (b) of a $300 \mu \mathrm{m}$ SRL.

susceptibility of the active material for a given carrier density $N$.

The power collected at ports \# 3 and \# 4 is $P_{3(4)}=\delta^{2}\left|E_{+(-)}\right|^{2}$, and it can be determined from eqs. (1-2). Fig. 7 shows the theoretical results for the detuning between the doublets, which are in good agreement with the experimental results reported in Fig.6. The doublet structure of the peaks is recovered when some small amount of gain in the SRL is included; if gain is not included, the spectral width of the peaks is large compared to their frequency splitting, and the doublet structure is smeared out into a single, asymmetrical peak.

The results for the transfer matrix to port \#4 show similar trends, and the transfer matrix analysis does not lead to dips on an spontaneous-emission noise background. The discrepancy is due to the fact that the transfer matrix analysis does not include the effects of spontaneous emission noise in the SRL cavity. Indeed, due to the (slight) gain in the SRL, the power collected at ports \#3 and \#4 in the absence of external light is the power due to spontaneous emission in the SRL, including amplification and attenuation in the path. In the absence of any reflecting element, light injected into the SRL through port \#1 would reach port \#3 only after being amplified or attenuated along the path, and no injected light would reach port \# 4; however, the power at port \# 4 would be reduced because of ASE suppression under light injection, thus leading to dips onto the ASE background. This is indeed the experimental situation, where the output waveguides have been reverse biased, and the explanation is confirmed by measuring plotting the envelope of the power collected at port \#4 for different bias currents in the SRL cavity (see Fig. 5): the dips cannot be seen for bias currents below $30.5 \mathrm{~mA}$ and being clearly visible above. It is worth remarking that this effect provides us with a precise way to measure the spectral dependence of the transparency current. 


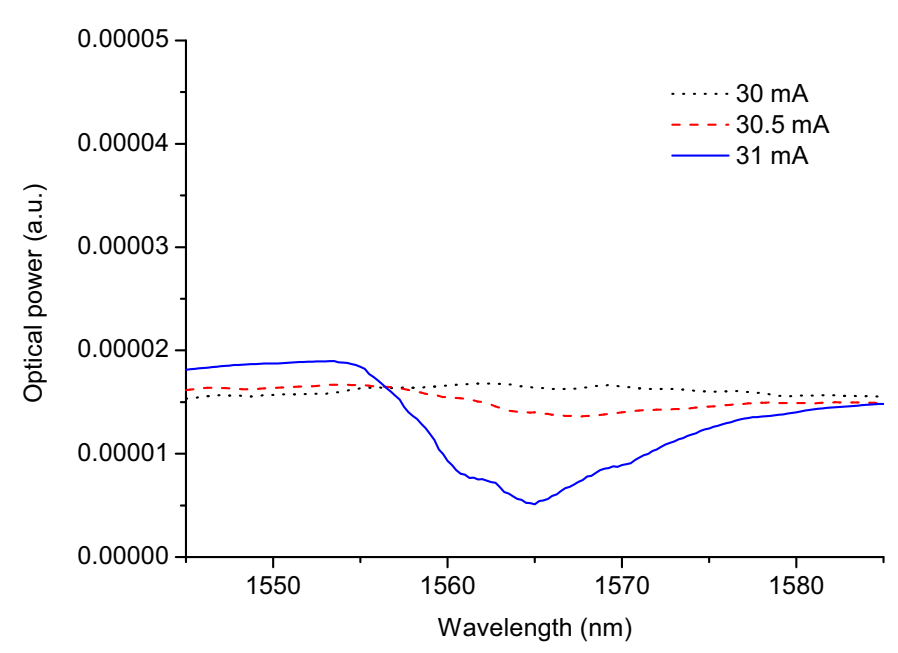

Figure 5. Envelope of the measured peaks from port \#4 for different SRL currents.

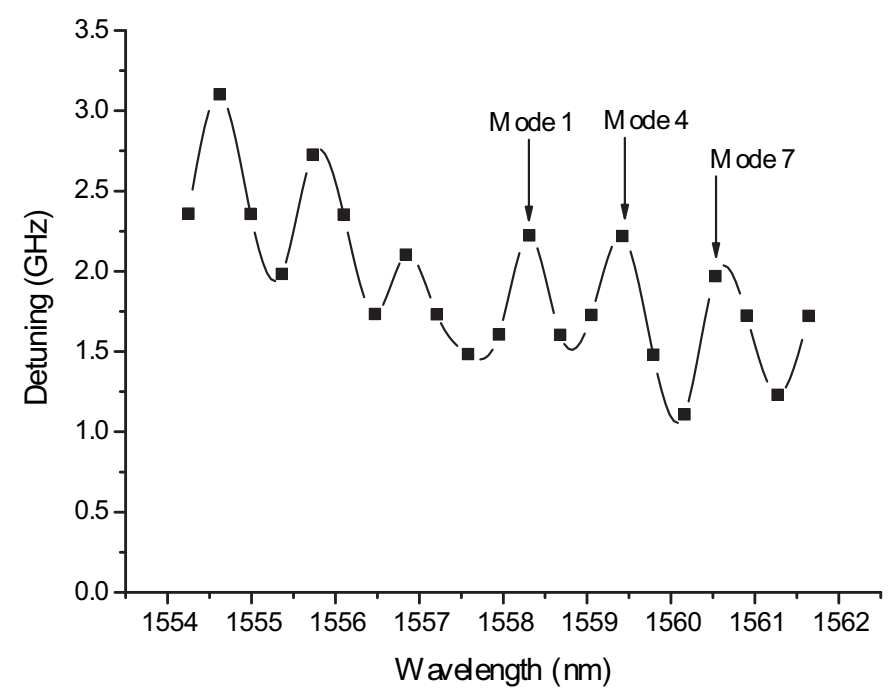

Figure 6. Measured detuning between doublets.

The modal selection can be explained by considering the modulation imposed by the cavity losses together with the red-shift of the gain peak with current as due to Joule heating of the laser junction. The modes of the device can be determined from (1) and (2) by requiring that a non-trivial solution exists in the absence of injected fields. This leads to the threshold condition

$$
\begin{aligned}
{[1} & \left.-\left(1-\delta^{2}\right) m(\omega)-\left(1-\delta^{2}\right)(1-m(\omega)) e^{i \theta}\right]^{2} \\
& =m(\omega)\left(\delta^{2} \sqrt{1-\delta^{2}} e^{i \theta}\right)^{2},
\end{aligned}
$$

which possesses two solution branches. This is due to the breaking of the circular symmetry imposed by the output coupler, ${ }^{6}$ which implies that pure $\mathrm{CW}$ and $\mathrm{CCW}$ states do not exist anymore due to the defect. In the limit of weak reflectivities and moderate coupling efficiency, the modal frequencies and threshold carrier densities of the device read

$$
\omega_{k}^{ \pm} \approx \frac{2 \pi k}{\tau_{R}} \mp \frac{\varepsilon}{\tau_{R}} \sqrt{1+\alpha_{k}^{2}} \sin \left(\pi k \frac{\tau_{F P}}{\tau_{R}}+\operatorname{atan} \alpha_{k}\right)
$$




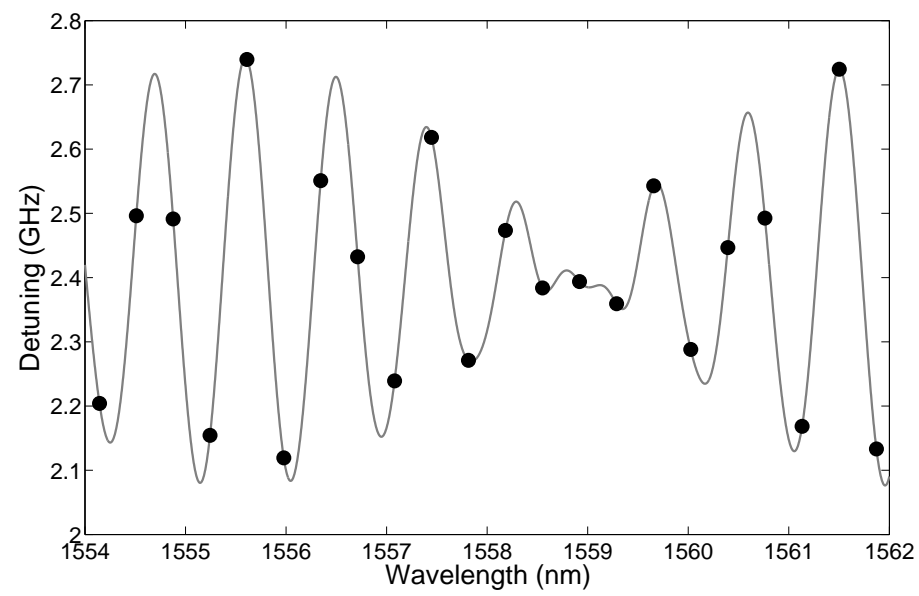

Figure 7. Theoretical detuning between doublets.

$$
N_{k}^{ \pm}=N_{k}^{t}+\frac{1}{g_{k}}\left[\alpha_{t o t} \mp \varepsilon \cos \left(\pi k \frac{\tau_{F P}}{\tau_{R}}\right)\right]
$$

where $\varepsilon=\delta^{2} \sqrt{r_{1} r_{2}}, \tau_{R(F P)}$ is the roundtrip time in the SRL (Fabry-Perot) cavity, $g_{k}, N_{k}^{t}$ and $\alpha_{k}$ are the differential modal gain, transparency carrier density and linewidth enhancement factor of the $k-t h$ SRL mode, and $\alpha_{t o t}=\alpha_{i} L_{R}-(1 / 2) \ln \left(1-\delta^{2}\right)$ are the total cavity losses. Since $\alpha_{k}$ is usually large $(\gtrsim 2-3)$, the maximum frequency splitting of the doublets almost coincides with their maximum threshold difference. Moreover, the threshold modulation for each of the two branches of solutions is out of phase. Hence, when the gain spectrum redshifts due to Joule heating, the system will jump from the minimum on one branch to the following minimum on the other branch. For circular SRL, where $L_{R} \geq 2 R$, this means a jump of $m=i n t\left[3 \tau_{R} / \tau_{F P}\right]$ modes of the SRL, which for the device considered yields $m=3$.

\section{CONCLUSIONS}

In summary, we have experimentally studied the resonances of SRL devices both below and above threshold by measuring the transfer properties of the device below threshold and the wavelength jumps that occur in connection with directional switching above threshold. A transfer matrix analysis allows us to successfully explain the transfer function measurements when amplified spontaneous emission in the SRL cavity is accounted for. The same transfer matrix analysis permits to determine the threshold condition for the laser modes, which split in two branches due to the symmetry breaking imposed by the output coupler and output waveguides. The frequency splitting and threshold difference between these two branches of solutions, together with the redshift of the material gain, explain the wavelength jumps displayed by the SRL above threshold.

\section{ACKNOWLEDGMENTS}

We acknowledge financial support from project IOLOS, FP6-2005-IST-5. S. B. acknowledges financial support from project TEC2006-13887-C05-03. A.P. and A.S. acknowledge Balear Government project QULMI PROGECIB-5A. A.S. acknowledges Ramón y Cajal program by Spanish MEC.

\section{REFERENCES}

1. Sorel, M., Giuliani, G., Scirè, A., Miglieria, R., Donati, S., and Laybourn, P. J. R., "Operating regimes og GaAs-AlGaAs semiconductor ring lasers: Experiment and model", IEEE J. Quantum Electron. 39, 1187 (2003).

2. Almeida, V.R., Barrios, C.A., Panepucco, R.R., Lipson, M., Foster, M.A., Ouzounov, D.G., and Gaeta, A.L., "All-optical switching on a silicon chip", Opt. Lett. 29, 2867 (2004). 
3. Hill, M. T., Dorren, H. J. S., de Vrie, T., Leijtens, X. J. M., den Besten, J. H., Smalbrugge, B., Oei, Y. S., Binsma, H., Khoe, G. D. and Smit, M. K., "A fast low-power optical memory based on coupled micro-ring laser", Nature 432, 206 (2004).

4. Booth, M.F., Schremer, A., and Ballantyne, J.M., "Spatial beam switching and bistability in a diode ring laser", Appl. Phys. Lett. 76, 1095 (2000).

5. Sorel, M., Laybourn, P. J. R., Giuliani, G. and Donati, S., "Unidirectional bistability in semiconductor waveguide ring lasers", Appl. Phys. Lett. 80, 3051 (2002).

6. D'Angelo, E.J., Izaguirre, E., Mindlin, G.B., Huyet, G., Gil, L., and Tredicce, J.R., "Spatiotemporal dynamics of lasers in the presence of an imperfect O(2) symmetry ", Phys. Rev. Lett. 68, 3702 (1992).

7. Siegman, A.E., Lasers, University Science Books, Mill Valley, CA, pp. 398-426 (1986). 